\title{
Terrain Mapping Camera-2 onboard Chandrayaan-2 Orbiter
}

\author{
Arup Roy Chowdhury*, Vishnukumar D. Patel, S. R. Joshi, A. S. Arya, \\ Ankush Kumar, Sukamal Paul, Dhrupesh Shah, Pradeep Soni, J. C. Karelia, \\ Minal Sampat, Satish Sharma, Sandip Somani, H. V. Bhagat, Jitendra Sharma, \\ Amitabh, K. Suresh, R. P. Rajasekhar, B. B. Bokarwadia, Mukesh Kumar and \\ D. N. Ghonia
}

Space Applications Centre, Indian Space Research Organisation, Ahmedabad 380 015, India

\begin{abstract}
The paper presents the design and development of Terrain Mapping Camera-2 (TMC-2) for Chandrayaan-2 including science objectives; system and sub-system configuration along with the realized performance of the camera; payload characterization; aspects related to data products, etc. TMC-2, onboard Chandrayaan-2 orbiter-craft is a follow-on of the Terrain Mapping Camera (TMC) onboard Chandrayaan-1. It operates in visible panchromatic band. It comprises three identical electro-optical chains aligned for three views $(-25,0$ and +25 degree) along track direction for generation of stereo images. It provides data with $5 \mathrm{~m}$ horizontal ground sampling distance to generate digital elevation model. TMC-2 based on the new configuration and sub-system designs has reduction in mass and power by more than $\mathbf{4 0 \%}$ compared to TMC, without compromising the performance.
\end{abstract}

Keywords: Digital elevation model, light transfer characteristics, relative spectral response, signal-to-noise ratio, stereo imaging, square wave response, terrain mapping camera-2.

TERrAin Mapping Camera-2 (TMC-2) is continuation of the $\mathrm{TMC}^{1-3}$ of Chandrayaan-1 mission for topographic coverage of the Moon. Chandrayaan-2 is an orbiterlander-rover mission. This is India's second Moon mission. Similar to TMC, TMC-2 payload is configured to provide panchromatic images $(0.4 \mu \mathrm{m}-0.85 \mu \mathrm{m})$, stereo triplets (fore, nadir and aft views) at $5 \mathrm{~m}$ spatial resolution from $100 \mathrm{~km}$ circular orbit around Moon for preparing detailed 3-D map or Digital Elevation Model (DEM) of the complete lunar surface. DEM derived from TMC-2 will be used for detail morphometric analysis and photometric correction of hyper-spectral data obtained from Imaging Infrared Spectrometer (IIRS) on the same platform. Although the system specification is same as

\footnotetext{
*For correspondence. (e-mail: arc@sac.isro.gov.in)
}

TMC, there are new designs and design changes in almost all of the sub-systems of TMC-2 to meet the challenging requirements of reduction in mass and power without compromising on the overall performance. TMC-2 based on the new configuration and sub-system designs has reduction in mass and power of more than $40 \%$ compared to TMC.

\section{Science objectives}

The major objectives of TMC-2 is systematic morphological and stratigraphic mapping of lunar features and estimation of their morphometric parameters for reconstruction of lunar geological evolutionary history and understanding the past volcanic and tectonic processes. Objectives are described in detail as given below:

- Study of lunar volcanism through systematic mapping of domes/cones in highland and mare regions and estimation of their rheological properties using morphometric parameters.

- Study of the craters in both polar regions through stratigraphic mapping could be attempted.

- Integrated analysis of TMC, IIRS and dual frequency SAR data will be carried out for comprehensive geological interpretation of various geological units/ terranes.

- Lunar reflectance studies over selected sites on Lunar surface will also be attempted.

The images captured by the TMC of Chandrayaan-1 mission have been found to be extremely useful as it returned orbital high resolution stereo images and the unprecedented view of the lunar surface at that time. This enabled the maiden three-dimensional mapping of a volcanic lava tube on Moon ${ }^{4}$, which could be used for habitability of base station in future. Further, TMC data helped in detailed morphometric/rheological studies of the lunar dome/cones for understanding evolutionary 
history of the volcanic regions ${ }^{5,6}$ and surface age estimation of geological units ${ }^{7}$, which is required for correlative study of different terranes (basins, craters, etc. distributed on near and far side of the Moon). TMC onboard Chandrayaan-1 mission has mapped about $40 \%$ of the lunar surface. Morphological mapping and stratigraphic correlation of lunar features require reasonable continuous coverage. This can be achieved by acquisition of TMC-2 datasets in the gap regions of TMC of Chandrayaan-1. It is also going to help in creating high-resolution global image mosaic and DEM. High spatial resolution TMC-2 ortho images $(5 \mathrm{~m})$ along with DEM $(10 \mathrm{~m})$ will be used for mapping morphological features, estimating morphometric parameters and determining the surface age through crater size frequency distribution (CSFD) method to study stratigraphic relationships among various geological features. Comprehensive understanding of the evolution of various mare basins and different terrains distributed in the near and far sides of the Moon can be attempted through morpho-stratigraphic mapping using data from TMC-2, compositional mapping using data from IIRS integrated with lunar gravity anomaly maps from other mission. In addition, Chandrayaan-2 Orbiter High Resolution Camera (OHRC) datasets could be used for few selected areas identified from the analysis of TMC and IIRS datasets. DEM generated using TMC-2 datasets could also be used for photometric correction of IIRS datasets.

\section{System configuration}

Various sub-system configurations were studied to meet challenging mass and power budget. A new system configuration is worked out to achieve mass reduction by modification of optical configuration. TMC configuration is based on common optics for all the three views. TMC2 optical configuration has individual lens assembly for each of the three views to achieve miniaturization. Due to reduction in the field-of-view requirements, optical design is simpler and has lesser mass than the TMC optical system. Other sub-systems of TMC-2, viz. detector head assembly, camera electronics and mechanical systems are also newly designed to ensure reduction in payload mass and power. Camera electronics is miniaturized using state of the art components like smaller field programmable gate array, micro-D connectors, etc. and single printed circuit board and common packaging schemes. Reduced packaging size and lighter materials are used for housing to further reduce size and mass of the system. Linear detector with $4 \mathrm{k}$ pixels, $7 \mu \mathrm{m}$ pixel size and optics with $140 \mathrm{~mm}$ effective focal length (EFL) provides the $5 \mathrm{~m}$ ground sampling distance (GSD) and $20 \mathrm{~km}$ swath from $100 \mathrm{~km}$ altitude. The camera is configured for imaging in the push broom-mode with three linear detectors in the image plane.

\section{Optical configuration}

The main goal of the optical design is to achieve the best image quality, both in terms of geometric and radiometric performance, having minimum package dimensions and mass. Both refractive and reflective optics-based options were considered but due to large dimension and mass, reflective option was not pursued. Refractive optics (being a viable option) is designed with focal length of $140 \mathrm{~mm}, F / 4$ and field-of-view of $\pm 6^{\circ}$. The optical assembly consists of seven spherical lens elements with a thermal filter at front. Thermal filter is insensitive to temperature gradients between the front and rear surfaces, and helps to reduce heat load to the powered elements. Suitable band-pass filter is placed near focal plane to meet spectral range requirement. Titanium barrels are considered to meet system specifications with required thermomechanical stability. The optical schematic is shown in Figure 1.

\section{Detector head assembly}

To reduce overall mass of TMC-2 payload, various design aspects are looked into critically towards the detector head assembly (DHA) miniaturization. Several configurations were worked out to reduce detector head proximity electronics, mechanical interfaces and thermal interfaces without compromising DHA electro optical performance and also maintaining compatibility with TMC. DHA is designed with the same detector, which was used in TMC. The detector is an active pixel sensor (APS) having 4000 pixels arranged linearly, having pixel size of $7 \mu \mathrm{m} \times 7 \mu \mathrm{m}$, external exposure control and internal 12 bit digitizer. The three DHAs are identical for the three views. Micro-D connectors are considered for DHA to camera electronics (CE) interfaces due to which

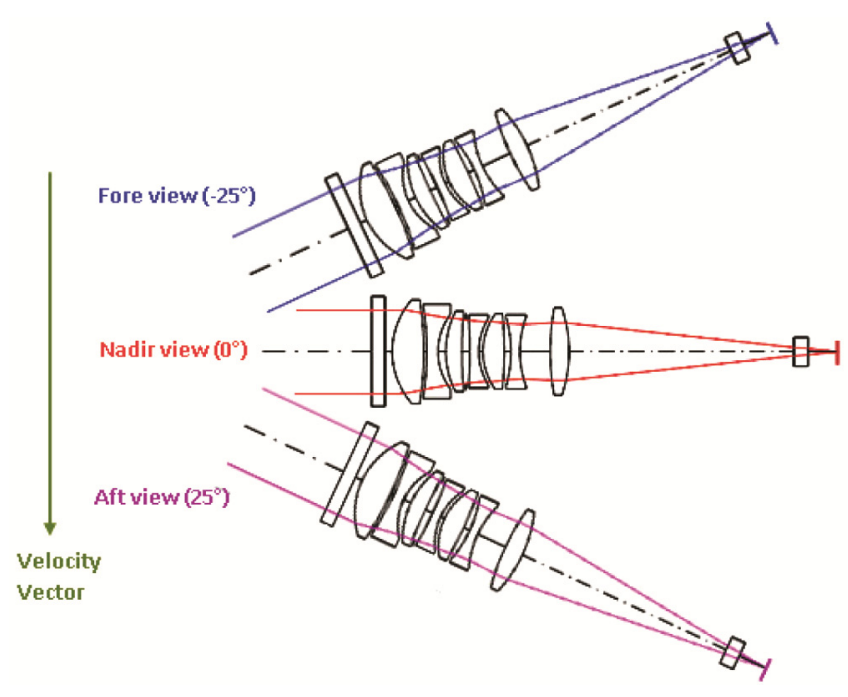

Figure 1. Optical schematic of terrain mapping camera-2. 


\section{RESEARCH ARTICLES}

considerable miniaturization is obtained in PCB size and mass. Since, detector power consumption is very less $(\sim 200 \mathrm{~mW})$, copper strip is replaced by copper foil for mass optimization while meeting thermal management requirements. Figure 2 shows a DHA.

\section{Camera electronics}

CE consists of detector proximity electronics (DPE), logic and control electronics (LCE) and power supply electronics (PSE). The detector is mounted in the DPE card (Figure 2). LCE (Figure 3) is miniaturized using state of art components. There are four values of exposures implemented in the electronics which are selectable by telecommand.

PSE (Figure 4) is configured for providing low noise regulated supply to all three LCEs and DHAs using main and redundant $\mathrm{DC}-\mathrm{DC}$ converter modules. $\mathrm{CE}$ mass is reduced to $0.45 \mathrm{~kg}$ from $1.8 \mathrm{~kg}$ of TMC.

\section{Mechanical systems}

Mechanical system comprises electro-optical module (EOM, optics and detector) structure, DHA mount and

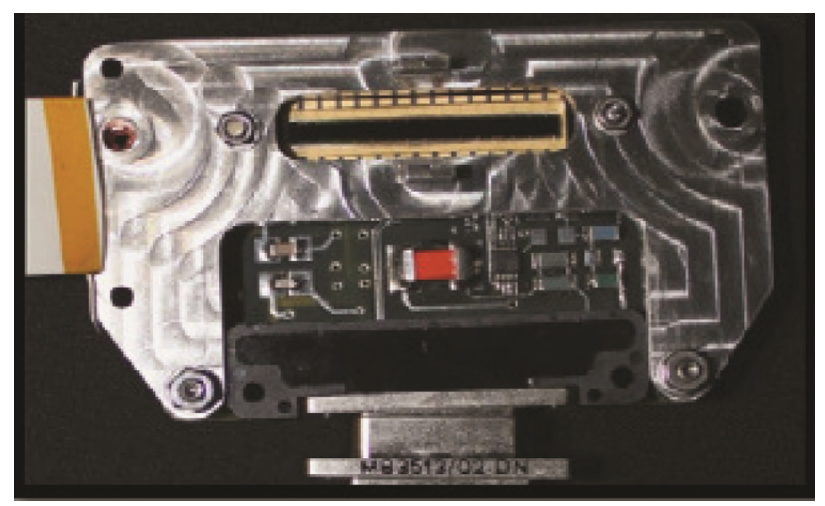

Figure 2. TMC-2 detector head assembly.

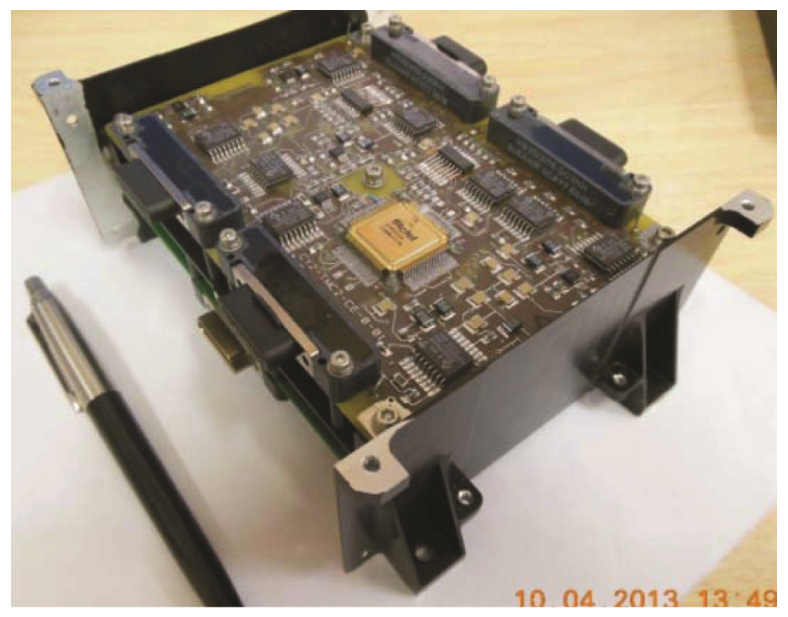

Figure 3. TMC-2 logic and control electronics card.
CE packages. EOM structure design is based on Titanium to meet thermo-mechanical requirements of lens assembly and focal plane, and miniaturized to meet overall mass requirement of the payload. Camera electronics package design is based on aluminium which provides common housing for LCE and PSE PCBs to save mass. It is mounted on the spacecraft deck.

Thermal design consists of passive thermal control techniques augmented with active auto-control heaters. EOM is mounted on anti-sun side and is covered with multi layer insulation. Heat is taken out through a metal strap running from detector to radiator.

\section{Flight model development}

TMC-2 flight model (FM) sub-systems were developed and performance was optimized followed by sub-system level test and evaluation (T\&E). The integrated payload (Figure 5) has successfully undergone system level T\&E as per Chandrayaan-2 environmental specifications. Payload performance throughout T\&E and post T\&E was found consistent.

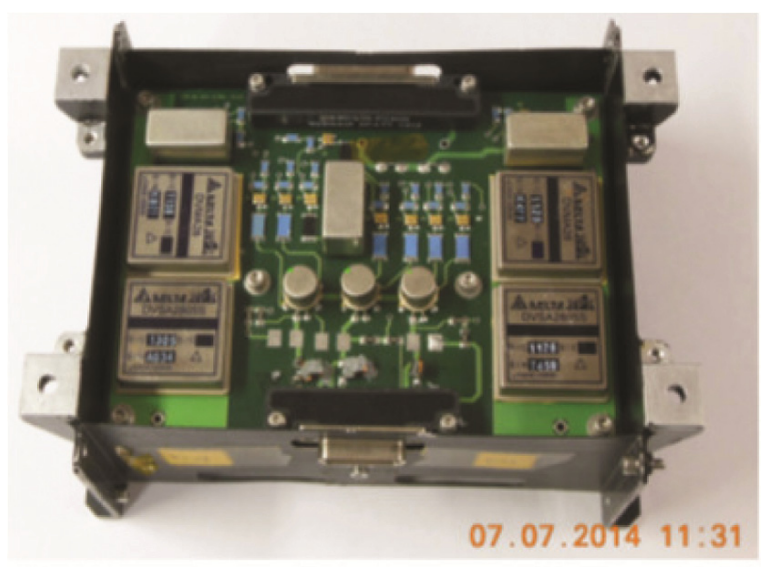

Figure 4. TMC-2 power supply electronics card.

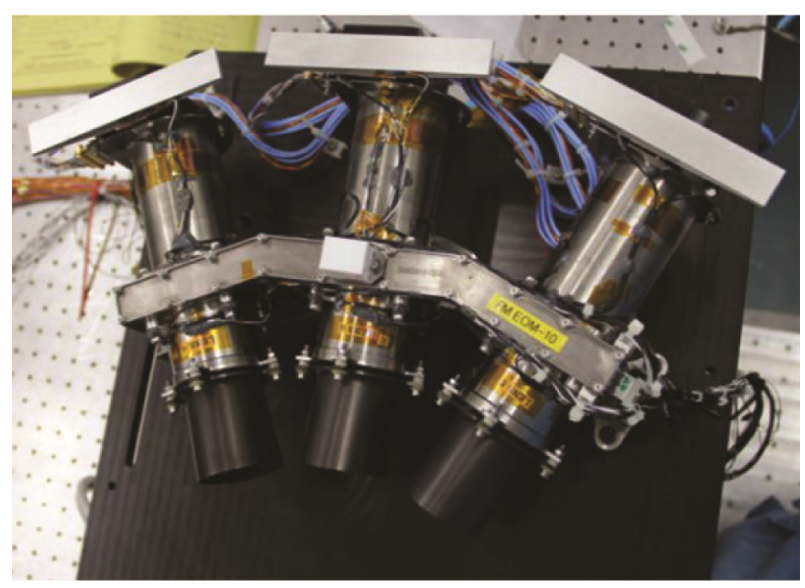

Figure 5. TMC-2 flight model.

CURRENT SCIENCE, VOL. 118, NO. 4, 25 FEBRUARY 2020 


\section{Payload characterization}

TMC-2 payload has been characterized for various performance parameters like: (i) signal-to-noise ratio (SNR) for noise performance; (ii) saturation radiance (SR) for dynamic range; (iii) linearity; (iv) square wave response (SWR) for image contrast performance, and (v) relative spectral response (RSR) for spectral band-width, etc. Figures 6-10 show performance of fore chain. Near saturation SNR is achieved $>500$ (Figure 6). Payload has been characterized for four programmable exposure settings. For the highest exposure setting, the SR is about $14 \mathrm{~mW} / \mathrm{cm}^{2} / \mathrm{sr} / \mu \mathrm{m}$ and for lowest exposure setting; it is about $28 \mathrm{~mW} / \mathrm{cm}^{2} / \mathrm{sr} / \mu \mathrm{m}$ (Figure 7). Payload response is

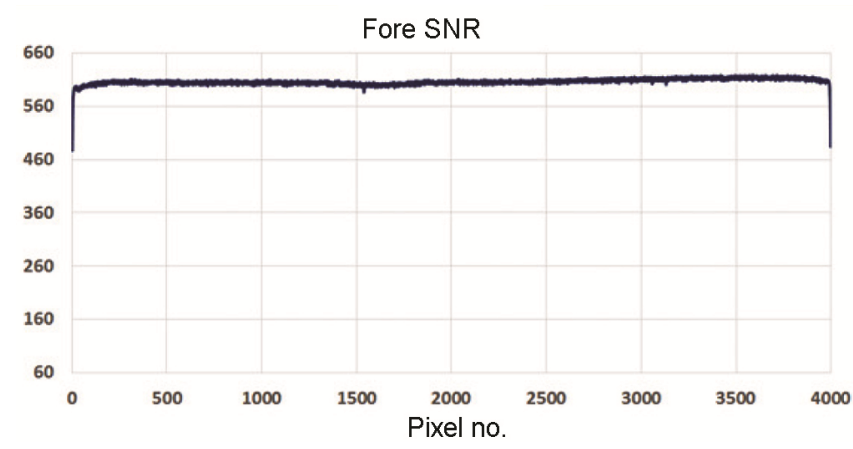

Figure 6. SNR profile of TMC-2 Fore channel.

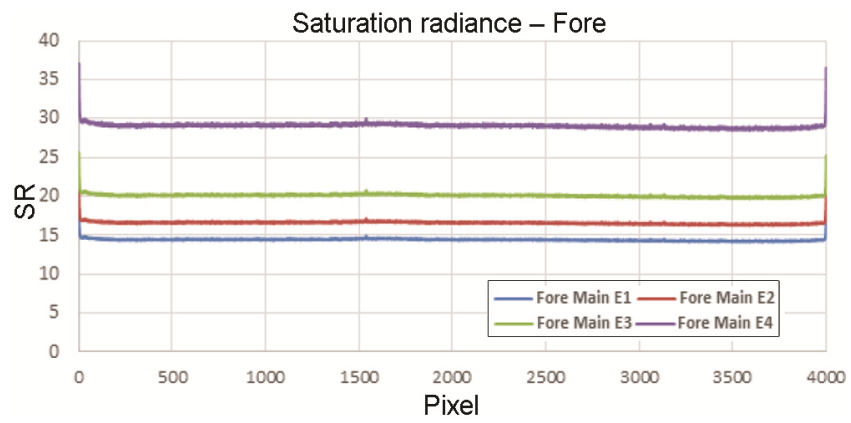

Figure 7. Saturation radiance $\left(\mathrm{SR}, \mathrm{mW} / \mathrm{str} / \mu \mathrm{m} / \mathrm{cm}^{2}\right)$ profiles for all four exposures for TMC-2 Fore channel.

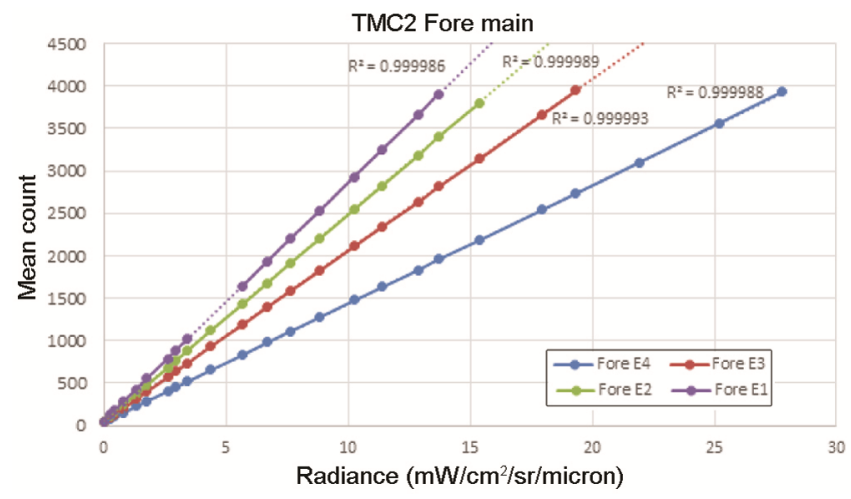

Figure 8. Linearity of TMC-2 Fore channel. highly linear and $R^{2}$ of the linear fit to the response is observed $>0.9999$. SWR is measured for three field positions of fore channel and at five different payload temperatures, namely $5^{\circ} \mathrm{C}, 10^{\circ} \mathrm{C}, 20^{\circ} \mathrm{C}, 30^{\circ} \mathrm{C}$ and $35^{\circ} \mathrm{C}$. SWR performance is nominal for different temperatures. (Figure 9). RSR is measured at different wavelengths in the range of 400-1100 $\mathrm{nm}$ (Figure 10). The other chains, i.e. aft and nadir chains also have nominal performance.

\section{Comparison between TMC (of Chandrayaan-1) and TMC-2 payload features}

Table 1 shows the comparison between TMC and TMC-2 in terms of various system parameters.

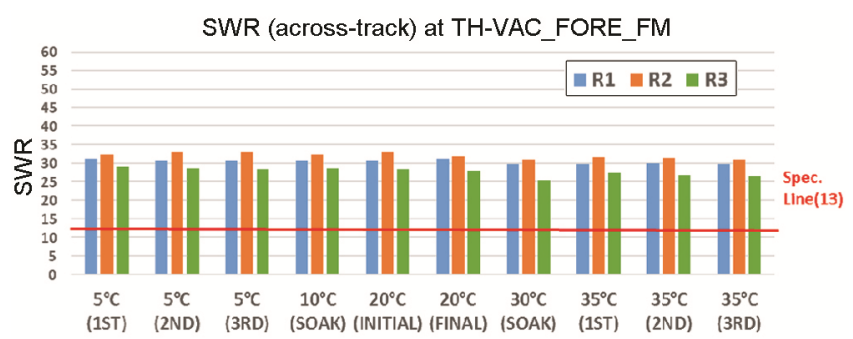

Figure 9. TMC-2 Fore channel square wave response.

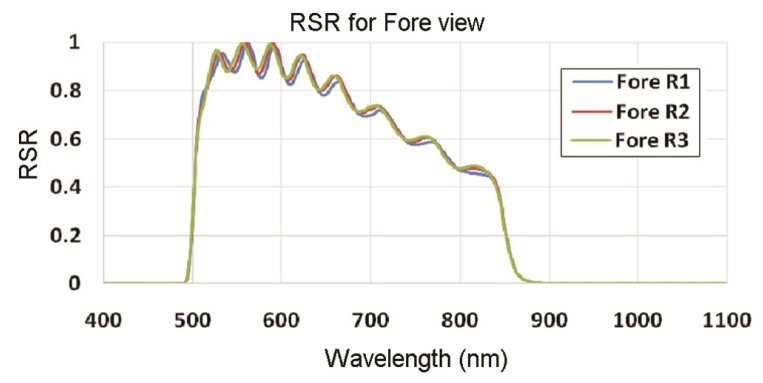

Figure 10. Relative spectral response of TMC-2 Fore channel.

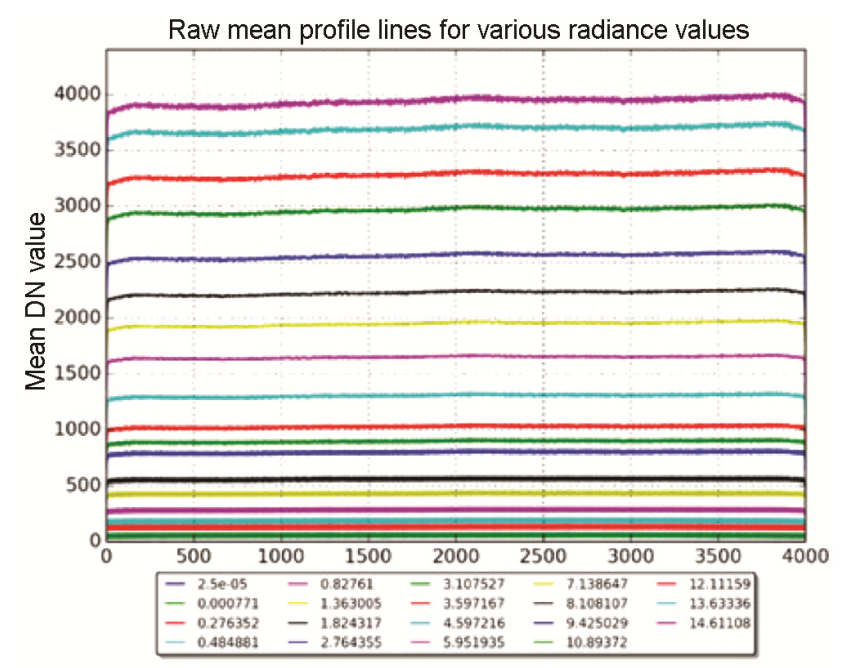

Figure 11. Plot of mean count versus pixel number of raw for Aft camera at E1. 
Table 1. System parameters of Terrain Mapping Camera - TMC and TMC-2

\begin{tabular}{lll}
\hline Parameter & \multicolumn{1}{c}{ TMC (Chandrayaan-1) } & \multicolumn{1}{c}{ TMC-2 (Chandrayaan-2) } \\
\hline Altitude $(\mathrm{km})$ & 100 & 100 \\
Spatial sampling $(\mathrm{m})$ & Along: 5 & Along: 5 \\
& Across: 5 & Across: 5 \\
Swath $(\mathrm{km})$ & 20 & 20 \\
Stereo mode & 3 -View along track; $\pm 25^{\circ}$ and $0^{\circ}$ & 3 -View along track, $\pm 25^{\circ}$ and $0^{\circ}$ \\
Spectral range $(\mu \mathrm{m})$ & $0.50-0.85$ & $0.50-0.85$ \\
Quantization $($ bits $)$ & 12 & 12 \\
Raw data rate per view $(\mathrm{Mbps})$ & 16.2 & 16.2 \\
Gain/exposure & 4 Each & $4-\mathrm{Exposures} \mathrm{and} \mathrm{single} \mathrm{gain}$ \\
Square wave response at Nyquist $(\%)$ & $>28$ & $>29$ \\
Signal-to-noise ratio & $\sim 68 @ 1.2 \mathrm{~mW} / \mathrm{cm}^{2} / \mathrm{str} / \mu \mathrm{m}$ & $>75 @ 1.2 \mathrm{~mW} / \mathrm{cm}^{2} / \mathrm{str}^{\circ} / \mu \mathrm{m}$ \\
& $>376 @ 14 \mathrm{~mW} / \mathrm{cm}^{2} / \mathrm{str} / \mu \mathrm{m}$ & $>500 @ 14 \mathrm{~mW} / \mathrm{cm}^{2} / \mathrm{str} / \mu \mathrm{m}$ \\
Raw power $(\mathrm{W})$ & 7.8 & 3.9 \\
Mass $(\mathrm{kg})$ & 6.3 & 3.5 \\
EOM $(\mathrm{mm})(Y \times P \times R)$ & $450 \times 254 \times 262$ & $280 \times 120 \times 440$ \\
\hline
\end{tabular}

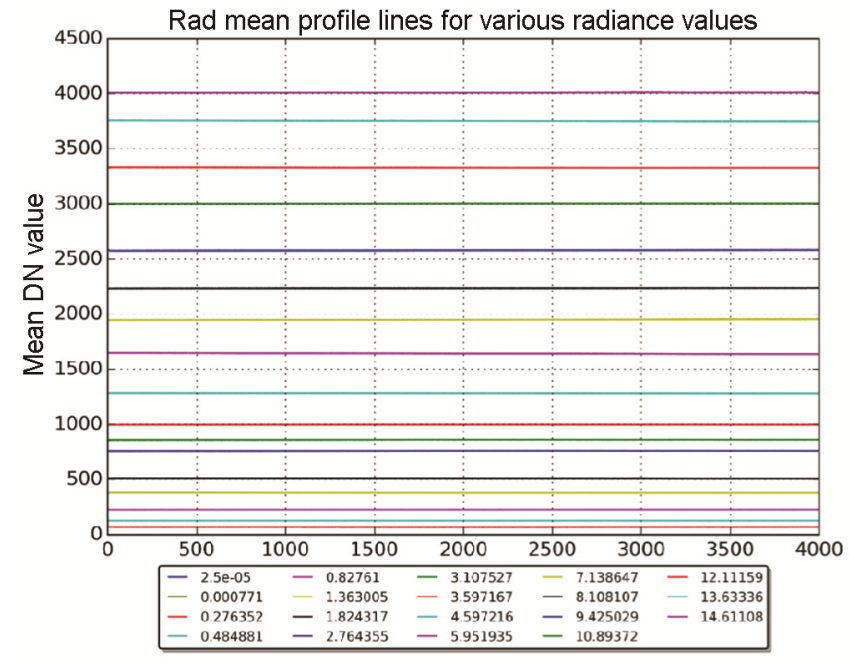

Figure 12. Plot of mean count versus pixel number of Rad for Aft camera at E1.

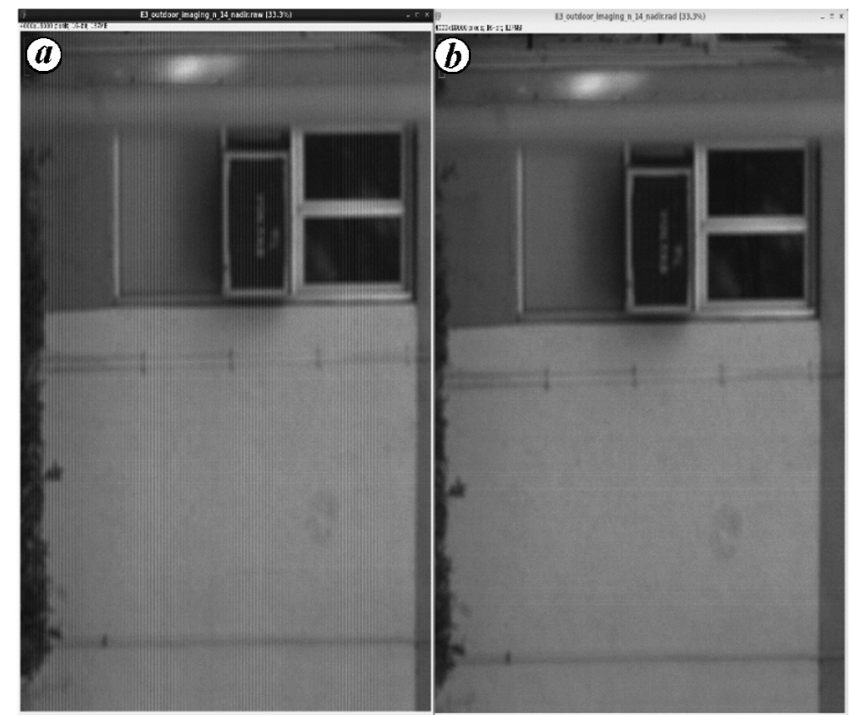

Figure 13. Images obtained during outdoor imaging by Nadir camera at exposure-4 (E4). Raw (a) and Rad (b) images.

\section{Overview of data products and payload characterization}

\section{Data products envisaged}

Three different types of data products are envisaged for TMC-2 payload of Chandrayaan-2. These products are level-0 (raw images), level-1 (radiometrically corrected and seleno tagged images) and level-2 (digital elevation model and ortho-images). Level-1 product generation for Fore, Nadir and Aft camera data involves detector-wise photo response non-uniformity (PRNU) correction which will be based on pre-launch laboratory calibration exercises; line and pixel drop out correction, noise characterization and tagging of corrected selenographic coordinates to each pixel. The level-2 product generation involves the extraction of DEM and corresponding co-registered ortho-images. The DEM generation process involves image matching between the suitable two or three images of the triplet, lunar control point (LCP) identification, resection, intersection, irregular DEM points generation and regular DEM and corresponding ortho-image generation using interpolation. The software pipeline for generating all these products in planetary data system (PDS 4) compliant format is developed, tested, evaluated and made ready at the Indian Space Science Data Centre (ISSDC), Bengaluru. Software for Data Products include level-0-2 (DEM and ortho-image), archival, browse and dissemination module, reference datasets and utilities to help ascertain radiometric and geometric accuracies.

\section{Light transfer characteristics experiment}

The light transfer characteristics (LTC) datasets were recorded in the lab for TMC-2 payload and used to analyse radiometric performance for getting normalization coefficients to correct for the PRNU of the detector array. 
RESEARCH ARTICLES

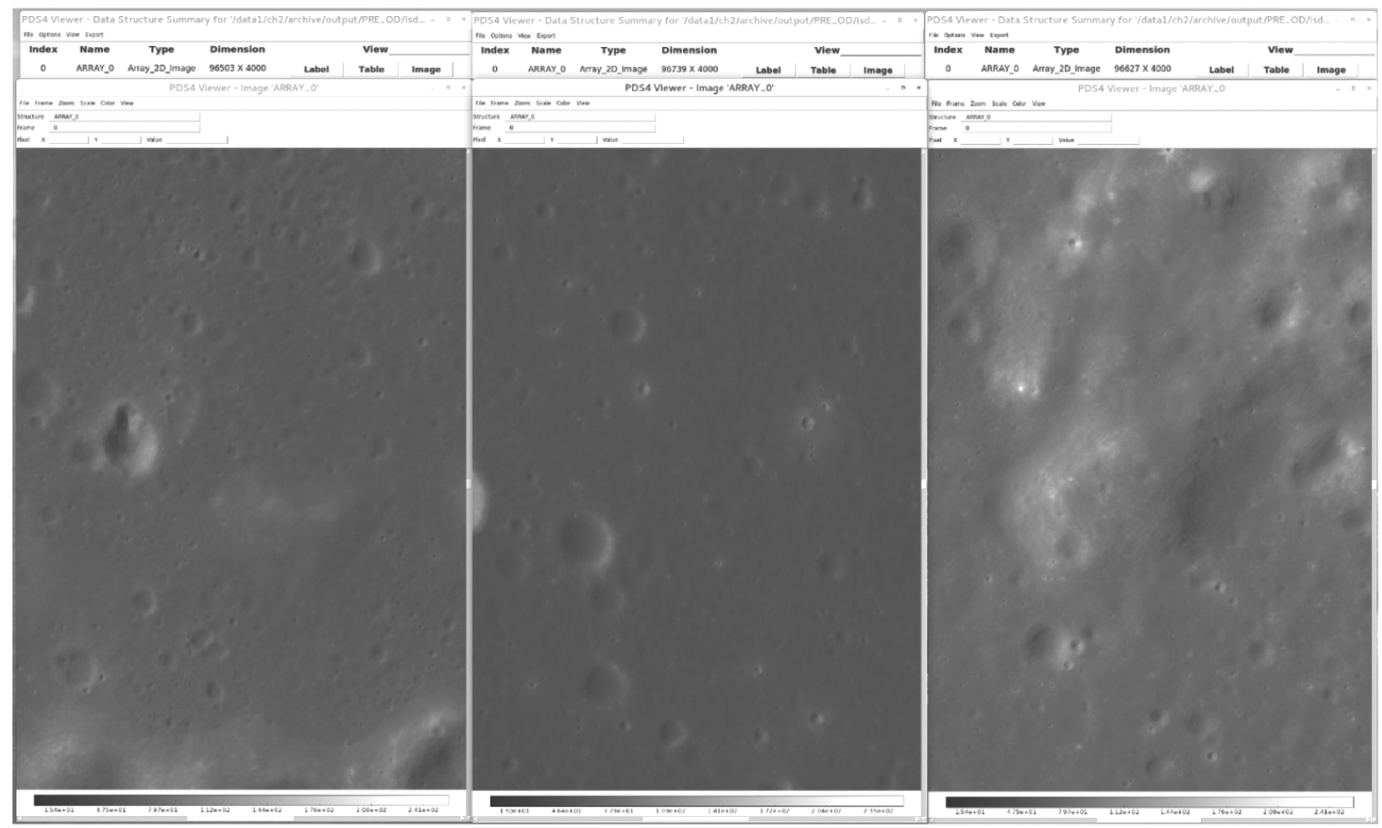

Figure 14. TMC-2 simulated Rad (level-1) images of Fore, Nadir and Aft channels.

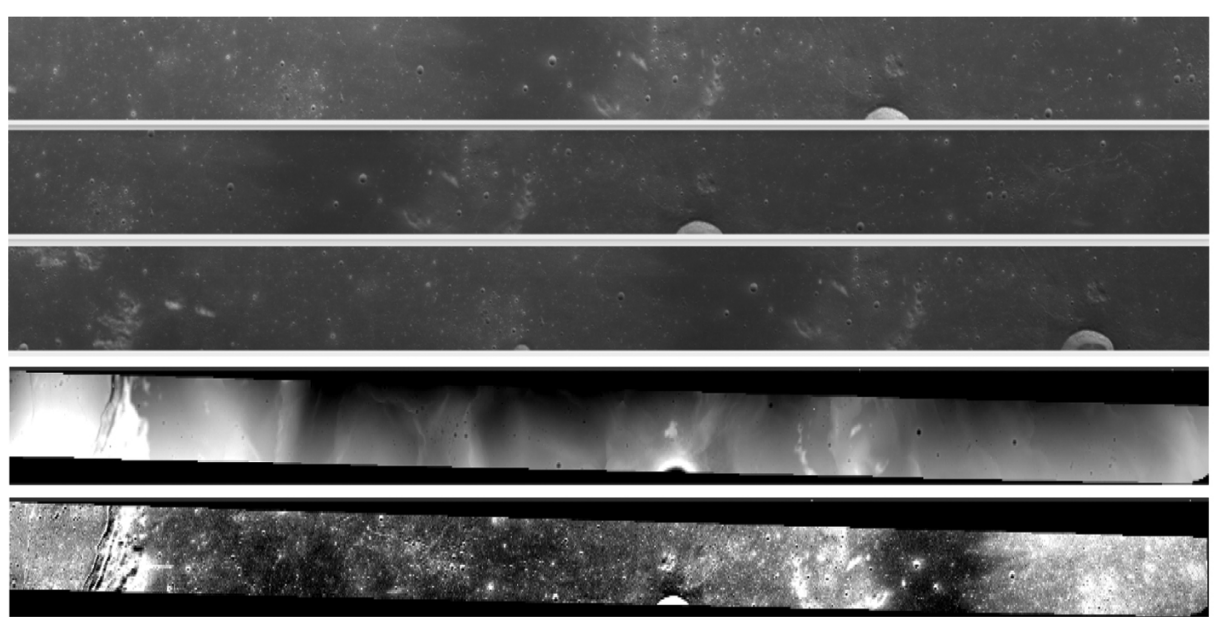

Figure 15. Simulated Fore, Aft and Nadir images of TMC-2 and corresponding digital elevation model and ortho-image (level-2) product.

Table 2. Detector array specifications related to light transfer characteristics experiment

\begin{tabular}{lccc}
\hline Camera & No. of pixels & No. of bad pixels & No. of bits per pixel \\
\hline Fore & 4000 & 0 & 12 \\
Aft & 4000 & 0 & 12 \\
Nadir & 4000 & 0 & 12 \\
\hline
\end{tabular}

The detector array specifications related to LTC experiment are provided in Table 2.

To have a qualitative understanding of the PRNU correction performed, mean counts at different levels were plotted against pixel values for both raw and look-up table applied data. Figure 11 shows the raw mean profile

CURRENT SCIENCE, VOL. 118, NO. 4, 25 FEBRUARY 2020 for various radiance values while Figure 12 shows the radiometrically corrected profile for Exposure-1 (E1).

\section{Outdoor acquired image}

The TMC-2 camera was operated for outdoor imaging for a target at distance around $30 \mathrm{~m}$ during day time. Figure 13 shows the raw and radiometrically corrected image of one of the outdoor images.

\section{Simulated Chandrayaan-2 data product}

Chandrayaan-1 TMC images and orbits were used to simulate the Chandrayaan-2 TMC-2 data product for testing 


\section{RESEARCH ARTICLES}

the data products generation software. The choice of Chandrayaan-1 TMC data is obvious because of the similarity in resolution of the cameras and the altitude of the platform for data acquisition. Figure 14 provides simulated Fore, Aft and Nadir Rad (level-1) images while Figure 15 provides a glimpse of triplet, DEM and orthoimage generated from the TMC triplet.

\section{Summary}

All the sub-systems of TMC-2 are newly designed to meet the challenging requirement of reduction in mass and power. Mass of TMC-2 payload is around $3.5 \mathrm{~kg}$ which is about $40 \%$ less with respect to TMC. Also, raw power requirement is reduced by $50 \%$ with respect to TMC. Optical performance, e.g. SWR and radiometric performance, e.g. SNR are better in TMC-2. SNR achieved during radiometric characterization is greater than 500 .

1. Kiran Kumar, A. S. and Chowdhury, A. R., Terrain mapping camera for Chandrayaan-1. J. Earth Syst. Sci., 2005, 114(6), 717-720.

2. Kiran Kumar, A. S. et al., Terrain mapping camera: a stereoscopic high-resolution instrument on Chandrayaan-1. Curr. Sci., 2009, 96 , 492-495.

3. Kiran Kumar, A. S. et al., The terrain mapping camera on Chandrayaan-1 and initial results. In 40th Lunar and Planetary Science Conference, Houston Texas, 2009, Abstract \#1584.
4. Arya, A. S., Rajasekhar, R. P., Guneshwar Thangjam, Ajai and Kiran Kumar, A. S., Detection of potential site for future human habitability on the Moon using Chandrayaan-1 data. Curr. Sci., 2011, 100, 524-529.

5. Arya, A. S., Rajasekhar, R. P., Amitabh, Gopala Krishna, B., Ajai and Kiran Kumar, A. S., Morphometric, rheological and compositional analysis of an effusive lunar dome using high resolution remote sensing data sets: a case study from Marius hills region. $A d v$. Space Res., 2014, 54, 2073-2086.

6. Arya, A. S. et al., Morphometric and rheological study of lunar domes of Marius Hills volcanic complex region using Chandrayaan1 and recent datasets. J. Earth Syst. Sci., 2018, 127, 70.

7. Arya, A. S. et al., Lunar surface age determination using Chandrayaan-1 TMC data. Curr. Sci., 2012, 102, 783-788.

ACKNOWLEDGEMENTS. We acknowledge with thanks the contributions of all colleagues involved in the realization of the instrument. The Sensors Development Area at Space Applications Centre (SAC) is the lead area responsible for overall design, development, testing, qualification and delivery of the instrument. The optical elements were fabricated at Laboratory for Electro-Optics Systems, Bengaluru. We gratefully acknowledge the support and guidance received from various areas of SAC and are thankful to the Director, SAC for the constant encouragement.

Received and accepted 27 August 2019

doi: $10.18520 / \mathrm{cs} / \mathrm{v} 118 / \mathrm{i} 4 / 566-572$ 